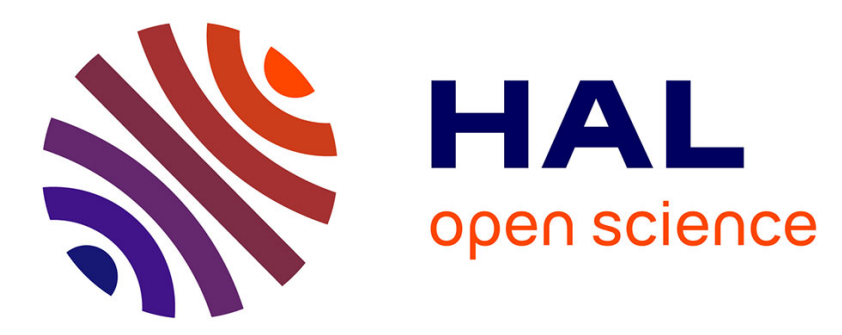

\title{
Digital, analogue, or redundant speedometers for truck driving: impact on visual distraction and usability
}

Mathilde Francois, Philippe Crave, François Osiurak, Alexandra Fort, Jordan Navarro

\section{- To cite this version:}

Mathilde Francois, Philippe Crave, François Osiurak, Alexandra Fort, Jordan Navarro. Digital, analogue, or redundant speedometers for truck driving: impact on visual distraction and usability. Applied Ergonomics, 2017, 65, pp. 12-22. 10.1016/j.apergo.2017.05.013 . hal-01677739

\section{HAL Id: hal-01677739 \\ https://hal.science/hal-01677739}

Submitted on 8 Jan 2018

HAL is a multi-disciplinary open access archive for the deposit and dissemination of scientific research documents, whether they are published or not. The documents may come from teaching and research institutions in France or abroad, or from public or private research centers.
L'archive ouverte pluridisciplinaire HAL, est destinée au dépôt et à la diffusion de documents scientifiques de niveau recherche, publiés ou non, émanant des établissements d'enseignement et de recherche français ou étrangers, des laboratoires publics ou privés. 
FRANCOIS, Mathilde, CRAVE, Philippe, OSIURAK, François, FORT, Alexandra, NAVARRO, Jordan, 2017, Digital, analogue, or redundant speedometers for truck driving: impact on visual distraction and usability, Applied Ergonomics, 65, Elsevier, pp. 12-22, DOI: 10.1016/j.apergo.2017.05.013

Digital, analogue, or redundant speedometers for truck driving: impact on visual distraction, efficiency and usability

\section{Abstract}

Existing literature does not draw conclusions as to which speedometer type is better for truck driving. A digital speedometer would be more beneficial when obtaining absolute and relative readings, while an analogue speedometer would be more efficient and less distracting when detecting dynamic speed changes. Redundant speedometers, which simultaneously present digital and analogue speedometers, appear increasingly in vehicles, but no information is available on their ergonomic qualities. This study compared three speedometers: digital speedometers, analogue speedometers, and redundant speedometers. This study compared the efficiency, usability and visual distraction measures for all three types of speedometers in a simulated truck driving setting. The task-dependant results were confirmed for the digital and analogue speedometer. The redundant speedometer combined the benefits of each type presented separately, which highlights interesting theoretical and applied implications.

\section{Keywords}

Speedometer design; truck interface; redundant displays 


\section{Introduction}

Speed is an essential piece of information that is necessary in order to driver in a proper manner. In addition to visual, auditory and vestibular cues, the speedometer on the dashboard provides an accurate speed reading for the driver, and provides assistance to the driver, so that the driver may properly assess the rate of speed by which they are travelling (Navarro, Mars \& Young, 2011). The design of the speedometer in trucks is critical for road safety, and is especially important for minimizing the need to glance to the side of the road. A misjudgement in the rate of speed is the second most common contributing factor to accidents where the truck driver was the cause (Volvo Trucks European Accident Research Safety Report, 2013). Speeding can lead to a rollover in a bend (particularly for concrete mixer trucks), jack-knifing when braking, or swinging out of control on slippery roadways (Volvo Trucks European Accident Research Safety Report, 2013). In the trucking industry, speedometers are mainly analogue. These speedometers consist of a moving indicator that slides along a scale. However, with the arrival of screen instrument clusters, the speedometer design is no longer constrained by technical reasons. Moreover, with the expansion of speed regulation systems, an increasing number of speed-related information appears on dashboards. This includes items such as adaptive speed limiters and cruise control systems, where the target speed is chosen by the driver. Truck designers are now facing new challenges, and require knowledge on the best way to present vehicle speed, in order to ensure fast, accurate and safe use of the truck.

In the human factors literature, studies focused mainly on two major speedometer types: analogue and digital speedometers. The efficiency of both speedometer types seems to be dependent on which task is performed. In an on-road study, Ishii (1980) reported that when drivers look at the digital speedometer, drivers look at it for less time than they look at an analogue speedometer, when engaging in normal driving conditions. More specifically, the amount of time that drivers using a digital speedometer spent looked at the speedometer was on average $70 \mathrm{~ms}$ shorter than when a driver looked at an analogue speedometer. Simmonds et al. (1981) conducted three studies with a total of 400 drivers, which studies compare different speedometer displays, including digital and analogue speedometers. Results showed that the digital speedometer was more accurate than the analogue speedometer when reporting the actual rate of speed. Howewer, when the speed value was compared to a limit, the accuracy was good for both types of speedometers. For both tasks, the digital speedometer reduced response times, increased usability, and was preferred by drivers. In addition, the number of subjective distractions were more balanced, depending on the driving environment. More specifically, the digital speedometer ranked as being less distracting in the on-road study, when compared to the simulator study. Likewise, Haller (1991) reported that a digital speedometer would be quicker to report a value that an analogue speedometer. Based on a complete review, Green (1988) argued that digital speedometers would be read more rapidly and accurately than analogue speedometers, and recommended that this type of speedometer be used by interface designers.

In contrast, in an on-road study, Walter (1991) reported that drivers using an analogue speedometer made more brake adjustments, and glances less at the speedometer than when using a digital speedometer. They added that even if the duration per glance was longer for the analogue speedometer, the duration of time spent with each driver's eyes off the road was shorter with the analogue speedometer than it was with the digital speedometer. The difference was noted as being on average, a difference of 1.5 seconds. Drivers also reported less stress and more control with the analogue speedometer (Walter, 1991). Similarly, Kiefer and Angell (1993) outlined a clear advantage for the analogue speedometer, when compared to the digital one. The analogue speedometer was found to be more effective for speed maintenance tasks, and it minimised visual distraction. This was the case even through there were a higher number of glances, as each glance was for a shorter duration. Finally, a more recent article presented three experiments that compared an analogue speedometer and a digital speedometer. Castro and Horberry (2004) reported that the digital display resulted in a slightly reduced response time when compared to the analogue speedometer. This occurred when it was being determined if the speed was over or under a limit, but this effect was reversed when the task was to detect changes in speed. With regard to subjective measures, Olaverri-Monreal et al. (2013) asked participants to prioritise different driving information for in-vehicle car displays. Both analogue and digital speedometers were listed as a first priority, but the analogue type was preferred by $71 \%$ of the participants.

The digital speedometer would be more appropriate when obtaining an absolute reading of the speed value (Green, 1988; Haller, 1991; Ishii, 1980; Simmonds et al., 1981) and a relative reading, such as comparing it to 
a target speed (Castro \& Horberry, 2004; Simmonds et al., 1981). However, reading a dynamic speed change would be more efficient and less distracting with an analogue speedometer than it could be with a digital speedometer (Castro \& Horberry, 2004; Kiefer \& Angell, 1993; Walter, 1991).

Another speedometer type that is appearing in new vehicles has, to the best of our knowledge, never been investigated in the literature discussing human factors. Redundant speedometers, which combine an analogue speedometer and a digital speedometer in the same instrument cluster, raise questions about whether the combined system can be processed efficiently. In fact, the same information is presented concurrently in two different forms. Even if cross-modal redundancy is mainly reported as being beneficial and a facilitator (e.g. Liu, 2001; Wickens \& Hollands, 2000), under these circumstances, the same information is displayed two times in a single modality. As a result of the cognitive load theory, researchers in instructional design (Sweller et al., 1998), argued that the same repeated information would degrade an individual's ability to process and comprehend, due to an increase in the external cognitive load. This principle, called redundancy effect, assumes that the different cues that are used simultaneously within the same modality would interfere with each other (Kalyuga et al., 2003; Wickens, 2002). Nonetheless, other researchers reported that a combination of text and symbols produced a better performance, than each one did when presented alone (Coury \& Pietras, 1989; Baber \& Wankling, 1992). Therefore, it is interesting to investigate if the redundant presentation of both speedometer types could either facilitate the completion of the task, such as drivers selecting the relevant information for a task, or produce an interference effect, where redundancy would degrade the ability to process information.

Most studies involving car drivers were conducted more than 20 years ago. Social changes (i.e. different relation to speed due to the strengthening of the regulation and road controls), technology changes (i.e. the expansion of digital types in the everyday life), and changes in term of context of use (e.g. accuracy needed to choose a cruise control target speed) could impact drivers' interaction with the speedometer. When compared to car drivers, truck drivers' eye distance to the instrument cluster is doubled to approximately $60 \mathrm{~cm}$, and the angle is increased. Researches showed that the difference of display positions between cars and trucks can influence a driver's glance behaviour (Larsson et al., 2017). Moreover, truck drivers spend most of their time on the road in a professional context. This can include up to 56 hours in any given work week (Bedinger et al., 2015). In addition, the difference in vehicle speed variation, due to vehicle weight, could impact their speed management and control mechanisms. Most of these assessments were conducted using analogue hardware, and it is essential to see if the benefits and tasks' specificities are preserved for screen instrument clusters. Moreover, studies were conducted based on basic tasks, such as reading the speed, comparing the speed to a target, or detecting a speed change. More complex use cases are missing in the literature, and may have different effect on drivers' behaviours and attitudes toward each speedometer type. For example, when displaying a road's speed limit on a traffic sign, the analogue scale might be processed more rapidly than when it is presented next to a digital display, which is benefiting from a spatial cue. In the Rasmussen's skillrules-knowledge model (1983), performance levels are based on different levels of information perception: signals, signs, and symbols. This difference in perception of information is due to context. More specifically, it is based on what information the user will use to achieve a given task. A digital speedometer would be processed at a sign perception level. Indeed, all tasks rely on rules, such as reading the value and comparing it to a value in memory, in order to determine if you are exceeding the speed limit. An analogue speedometer would be processed as a sign to report a speed value or compare it to a target speed. However, the ability to detect a speed change would benefit from signals that are provided by the continuous sensory-motor aspect associated with the movement of the pointer. The completion of this task would then rely on a skill-based behavioural level. Tasks using these different levels are required, in order to consider all processing aspects associated with each speedometer type.

This study compared three speedometers (digital, analogue and redundant) on efficiency, usability and visual distraction measures for truck driving. Speedometers were presented on a screen instrument cluster and assessed on contemporary use cases, such as comparing the truck's speed against the road's speed limit sign. The first objective was to update the existing literature on digital and analogue speedometers that have screen 
dashboard uses, and to examine the validity of the findings for truck drivers. The second objective was to evaluate the distraction, efficiency, and usability of a redundant speedometer, as opposed to the more traditional digital and analogue speedometers. Finally, the third objective was to assess and compare the three speedometer types while using speed management systems, such as cruise control, adaptive speed limiters, and the displaying of the road's speed limit.

\section{Material and methods}

\subsection{Participants}

A group of 18 trucks drivers took part in the experiment. All of the truck drivers were men, with a mean age of $42(S D=5.5)$. All participants held valid truck driving licences, with an average holding period of 16 years $(S D=8.8)$. Most participants drove a truck several times a month $(78 \%)$. All reported normal or corrected-tonormal vision and hearing. When driving a car, $67 \%$ of the participants used an analogue speedometer, $28 \%$ a digital speedometer, and 5\% used a redundant speedometer. When driving a truck, $78 \%$ of the participants used a digital speedometer, and $22 \%$ used either an analogue or a digital speedometer, depending on which truck the participant was driving. None of the participants regularly drove a truck with a redundant speedometer. Written informed consent was obtained from each participant.

\subsection{Equipment}

A fixed-base medium-fidelity driving simulator was used. The simulator was composed of a truck seat, twothirds of a real dashboard, and a 65 inch plasma screen that used Oktal SCANeR ${ }^{\mathrm{TM}}$ for traffic scenario display and truck modelling. Original accelerator pedal, brake pedal and a steering wheel from a Renault Trucks $\mathrm{T}$ were used. A highway environment was used, complete with simulated random traffic around the vehicle. A 15.4 inch screen was located in place of the instrument cluster behind the steering wheel, in order to display stimuli. The height of the screen was $332 \mathrm{~mm}$, the width of the screen was $207 \mathrm{~mm}$, and the resolution was 1280x800. A binocular head-mounted eye tracker was used to capture eye gaze (Tobii Glasses 2; scene camera resolution: 1920x1080; eye camera tracking frequency: $50 \mathrm{~Hz}$ ). Gaze raw data were filtered using the Tobii I-VT fixation filter configured so that short fixations were not discarded (Olsen, 2012).

\subsection{Material}

Speedometers. The digital speedometer was a two digit display (font: Myriad Pro; height: 18mm; Fig. 1-1a). The $\mathrm{km} / \mathrm{h}$ unit was displayed in lower case next to the digits (font: Myriad Pro; height: $5 \mathrm{~mm}$ ). The refresh rate of the digits was $1.25 \mathrm{~Hz}$ (refresh rate of the screen: $60 \mathrm{~Hz}$ ). The analogue speedometer was composed of a semi-circular fixed scale (width: $100 \mathrm{~mm}$, height: $50 \mathrm{~mm}$ ) graduated inside (length: $3 \mathrm{~mm}$ ) and marked outside all $10 \mathrm{~km} / \mathrm{h}$ from 0 and $120 \mathrm{~km} / \mathrm{h}$ (font: Myriad Pro; height: $5 \mathrm{~mm}$; Fig. 1-1b). The moving pointer was a noncomplete needle pointing the scale (10mm long, attached to the scale). The $\mathrm{km} / \mathrm{h}$ unit was displayed in small print at the beginning of the scale (font Myriad Pro; height: $3 \mathrm{~mm}$ ). For the redundant speedometer, the digital speedometer was presented at the centre of the analogue speedometer (Fig. 1-1c). All speedometers were presented centred in upper half of the screen. Decision on the graphic characteristics of the three speedometers have been taken considering existing fully reconfigurable clusters in cars (e.g. the needle is often cut off to display something inside), and the existing human factors knowledge (e.g. scale should be numbered in increments of 10; Green, 1988).

(insert Fig.1)

Tasks performed. The participants were required to perform a primary driving task which was to follow a red car on a highway. Moreover, each speedometer was tested using three different reading types: an absolute reading, a relative reading, and a dynamic reading. Three tasks, without additional speed information (i.e. pure driving) were proposed:

- Task1 (absolute reading): 'read the speed value' (answer: number)

- Task2 (relative reading): 'determine if the speed is under or over $50 \mathrm{~km} / \mathrm{h}$ ' (answer: plus or minus) 
- Task3 (dynamic reading): 'determine if the speed is increasing or decreasing' (answer: increase or decrease)

Moreover, three tasks implying additional speed information (i.e. driving with speed regulation systems) were proposed (Fig. 1-2):

- Task4 (absolute reading): 'read the speed value' (answer: number)

- Task5 (relative reading): 'determine if the speed is under or over the road speed limit (displayed on the speedometer)' (answer: plus or minus)

- Task6 (dynamic reading): 'determine the moment when the speed reach the cruise control target speed (as in a situation of pressing the 'resume' button of the cruise control)' (answer: top)

The instrument cluster remained black while driving. Drivers could maintain vehicle speed by adjusting the distance from the red car. The speedometer was displayed only during tasks and only from the start of the task until the driver provided an answer. Thus, it avoided having the driver look at the speedometer before the task. Moreover, the speed value displayed during each event was not related to the vehicle's current speed. By proceeding in this manner, reading times were entirely based on the type of display and the speed values that were being displayed could be controlled.

For the task 1, 4 stimuli were proposed ( 2 randomly chosen between 31 and $44 \mathrm{~km} / \mathrm{h}$ and 2 between 51 and 64 $\mathrm{km} / \mathrm{h}$ ). For the task 2, 8 stimuli were proposed ( 4 randomly chosen between 41 and $48 \mathrm{~km} / \mathrm{h}$ and 4 between 52 and $59 \mathrm{~km} / \mathrm{h}$ ). For the task 3,8 stimuli were proposed ( 2 decelerations and 2 accelerations with an initial speed randomly chosen between 36 and $41 \mathrm{~km} / \mathrm{h}$, and 2 decelerations and 2 accelerations with an initial speed randomly chosen between 56 and $61 \mathrm{~km} / \mathrm{h}$ ). For the task 4,4 stimuli were proposed ( 2 randomly chosen between 31 and $44 \mathrm{~km} / \mathrm{h}$ and 2 between 51 and $64 \mathrm{~km} / \mathrm{h}$ ). Additional information displayed was the road speed limit sign and the speed limiter target speed. For the task 5, 8 stimuli were proposed ( 2 randomly chosen between 41 and $48 \mathrm{~km} / \mathrm{h}$ and 2 chosen between 52 and $59 \mathrm{~km} / \mathrm{h}$ with the road speed limit at $50 \mathrm{~km} / \mathrm{h}$, and 2 randomly chosen between 61 and $68 \mathrm{~km} / \mathrm{h}$ and 2 chosen between 72 and $79 \mathrm{~km} / \mathrm{h}$ with the road speed limit at $70 \mathrm{~km} / \mathrm{h}$ ). Additional information displayed was the road speed limit sign. For the task 6,4 stimuli were proposed ( 1 slow acceleration $-0.21 \mathrm{~m} / \mathrm{s}^{2}$ - and 1 fast acceleration $-1.26 \mathrm{~m} / \mathrm{s}^{2}-$ with an initial speed randomly chosen between 38 and $42 \mathrm{~km} / \mathrm{h}$ and a cruise control target at $52 \mathrm{~km} / \mathrm{h}$, and 1 slow acceleration $-0.21 \mathrm{~m} / \mathrm{s}^{2}-$ and 1 fast acceleration $-1.26 \mathrm{~m} / \mathrm{s}^{2}$ - with an initial speed randomly chosen between 58 and $62 \mathrm{~km} / \mathrm{h}$ and a cruise control target at $72 \mathrm{~km} / \mathrm{h}$ ).

\subsection{Procedure}

Before the experimental phase, participants were informed of the details of the study, and each participant completed a consent form. After these steps were completed, the eye tracking device was positioned and calibrated. The participants were given a 10 minute training, in order to familiarise each participant with all of the speedometers and each of tasks. For each test drive, participants were required to follow a red car on a highway. The question drivers had to answer was stated by the experimenter before departure. During most of the experiment, the information cluster remained black. Every 6 to 8s (randomly) a sound announced that the speedometer was about to be displayed (Fig. 2). Thereafter, the driver could then give his answer aloud, which would result in a removal of the display. The experiment was composed of three test drives, each of which corresponded to the three speedometer types. The three test drives each containing the six tasks. A usability questionnaire was proposed after each test drive (cf. data acquisition and analysis section). At the end of the experiment, participants were asked to report their preferences, by providing a list that ordered their preferences, and by assigning grades to each preference.

Each participant was tested individually, and each participant experienced all conditions. The order in which the experimental condition were presented was counterbalanced by following Latin squares, crossing the speedometer types, the task group (i.e. simple driving or driving with additional information), and the tasks. The presentation of the stimuli was randomly arranged for each session. The total test duration was approximately one hour. 


\subsection{Data acquisition and analysis}

Visual distraction was analysed by calculating the mean number of glances away from the road and the mean total off-road glance duration. The glance times were computed from the time the stimulus was displayed until the time the driver started to give their answer. The efficiency was assessed through task completion times, measured as the time in milliseconds between the display of the stimulus and the answer of the participant. In addition, the efficiency was assessed using accuracy scores, measured as the absolute distance between the driver's answer and the real value displayed). Perceived usability was examined through a questionnaire composed of five 5 points Likert scales, each of which corresponded to the five constructs of usability, as defined by Nielsen (1994). The five constructs are learnability, efficiency, memorability, errors, and satisfaction. The goal of this questionnaire created for the study, was to offer a consistent subjective measure which could be compared to each of the three speedometers for each construct. In addition, the goal was to have it easy to follow and easy to respond to. Each item consisted of an affirmation based on the construct definition, and was associated with a Likert scale from 1 meaning: 'totally disagree', to 5, which meant: 'totally agree' (Appendix 1). Finally, driver satisfaction was measured at the end of the experiment. The order of preference (from 1 to 3) was collected orally. One represented the most satisfying speedometer, and three represented the least satisfying speedometer. The satisfaction score was collected for each speedometer using an 11 point Likert scale. In this scale, zero meant 'not satisfying at all', and ten meant 'very satisfying').

To compare the means of the three matched groups that corresponded to the three speedometer types, one-way repeated ANOVA measures were performed for each task, and were based on total glance duration and task completion times (within-subject factor manipulated: speedometer with three modalities: digital, analogue and redundant). To compare the three speedometers on the paired tasks (i.e. same question with and without additional speed information), $3 \times 2$ repeated measures ANOVA were performed on the glance and task completion times. The within-subject factors manipulated were: speedometers (digital, analogue, redundant) and tasks (absolute/relative reading without additional information: Task 1/Task 2, absolute/relative reading with additional information: Task 4/Task 5). The tasks 3 and 6 were not considered as paired tasks because the questions asked were different. Values apart from the mean value minus or plus two standard deviations were discarded from the data analysis (less than 5\% of the data). Tukey HSD post-hoc tests were leaded to determine significant differences of means between groups of the ANOVA. Non-parametric Wilcoxon tests for paired samples and Friedman tests were performed for the number of glances, accuracy scores, usability questionnaire and satisfaction measures. For the usability questionnaire, single item scores were computed to measure each of the five constructs.

\section{Results}

\subsection{Visual distraction}

(insert Fig.3)

(insert Fig.4)

For the task 1, the main effect of speedometer type was significant $\left(F(2,34)=102.21, p<.001, \eta_{\mathrm{p}}{ }^{2}=0.84\right)$ indicating a higher total glance duration (Fig. 3) with the analogue speedometer (1071ms) than with the digital (368ms; $p<.001)$ and the redundant speedometer (462ms; $p<.001)$. The same results were found for the task $\mathbf{4}$, with a significant effect of speedometer $\left(F(2,34)=140.92, p<.001, \eta_{\mathrm{p}}{ }^{2}=0.89\right)$ showing a higher total glance duration with the analogue speedometer $(1300 \mathrm{~ms})$ than with the digital $(456 \mathrm{~ms} ; p<.001)$ and the redundant speedometer $(522 \mathrm{~ms} ; p<.001)$. The $3 \times 2$ repeated-measures ANOVA revealed significant main effects of the speedometer $\left(F(2,34)=181.73, p<.001, \eta_{\mathrm{p}}{ }^{2}=0.91\right)$, of the tasks $\left(F(1,17)=16.69, p<.001, \eta_{\mathrm{p}}{ }^{2}=0.50\right)$, and the two-way interaction $\left(F(2,34)=4.05, p=0.027, \eta_{\mathrm{p}}^{2}=0.19\right)$. Post-hoc tests showed that, with the analogue speedometer, the total glance duration was significantly lower for task 1 than for task 4 (respectively $1071 \mathrm{~ms}$ and $1300 \mathrm{~ms} ; p<0.01$ ).

For the task 2, the one-way repeated measures ANOVA showed a significant effect of the speedometer $\left(F(2,34)=63.69, p<.001, \eta_{\mathrm{p}}{ }^{2}=0.79\right)$ highlighting that the analogue speedometer $(686 \mathrm{~ms})$ implied higher total 
glance duration than the digital (326ms; $p<0.01)$ and the redundant speedometer $(457 \mathrm{~ms} ; p<0.01)$. Similarly, the redundant speedometer implied longer glance duration than the digital speedometer $(p<0.01)$. These results were not found for the task 5, for which no significant differences were found between the speedometers regarding the total glance duration $(742 \mathrm{~ms}$ for the digital, $722 \mathrm{~ms}$ for the analogue, and $779 \mathrm{~ms}$ for the redundant; $F(2,34)=0.84, p=0.439)$. The $3 \times 2$ repeated-measures ANOVA revealed significant main effects of the speedometer $\left(F(2,34)=20.28, p^{<.001,}, \eta_{\mathrm{p}}{ }^{2}=0.54\right)$, of the tasks $\left(F(1,17)=56.04, p<.001, \eta_{\mathrm{p}}{ }^{2}=0.77\right)$, and the two-way interaction $\left(F(2,34)=24.57, p<.001, \eta_{\mathrm{p}}{ }^{2}=0.59\right)$. Post-hoc tests showed that the total glance duration was significantly higher for the task 5 than for the task 2 for with the digital speedometer (Task 2: 326ms, Task 5: 742ms; $p<.001$ ) and with the redundant speedometer (Task 2: 457ms, Task 5: 779ms; $p<.001$ ).

For the task 3, the effect of speedometer was significant $\left(F(2,34)=36.67, p<.001, \eta_{\mathrm{p}}{ }^{2}=0.68\right)$. The total glance duration away from the road was higher with the digital speedometer $(877 \mathrm{~ms})$ than with the analogue $(563 \mathrm{~ms}$; $p<.001)$ and the redundant speedometer $(633 \mathrm{~ms} ; p<.001)$.

For the task 6, no significant difference of total glance duration was found between the three speedometers (4604ms for the digital, $4320 \mathrm{~ms}$ for the analogue, and $4213 \mathrm{~ms}$ for the redundant; $F(2,34)=1.45, p=0.249$ ). However, the digital speedometer implied significantly higher number of glances than the analogue speedometer (respectively 5.04 and 4.26 glances; $Z=3.419, p<.001, r=0.57$; Fig. 4) and the redundant speedometer (4.21 glances; $Z=3.067, p=0.002, r=0.51$ ).

\subsection{Efficiency}

(insert Fig.5)

(insert Fig.6)

Objectives measures of efficiency were collected: task completion times (Fig. 5) and accuracy (i.e. absolute distance between the driver's answer and the value displayed; Fig. 6). For the task 1, the main effect of speedometer was significant $\left(F(2,34)=177.85, p<.001, \eta_{\mathrm{p}}{ }^{2}=0.91\right)$ indicating longer completion times with the analogue speedometer $(1442 \mathrm{~ms})$ than with the digital $(795 \mathrm{~ms} ; p<.001)$ and the redundant speedometer $(831 \mathrm{~ms} ; p<.001)$. The same results were found for the task 4 : significant effect of speedometer $\left(F(2,34)=151.33, p<.001, \eta_{\mathrm{p}}^{2}=0.90\right)$ showing a higher task completion time with the analogue speedometer $(1703 \mathrm{~ms})$ than with the digital $(874 \mathrm{~ms} ; p<.001)$ and the redundant speedometer $(879 \mathrm{~ms} ; p<.001)$. The $3 \times 2$ repeated-measures ANOVA comparing the three speedometers on the task 1 and 4 revealed significant main effects of the speedometer $\left(F(2,34)=208.04, p<.001, \eta_{\mathrm{p}}{ }^{2}=0.92\right)$, the tasks $\left(F(1,17)=40.75, p<.001, \eta_{\mathrm{p}}{ }^{2}=0.71\right)$, and the two-way interaction $\left(F(2,34)=12.01, p<.001, \eta_{p}^{2}=0.41\right)$. Tukey HSD post-hoc test showed that the task completion times were statistically significantly lower for task 1 than for task 4 with the analogue speedometer (respectively $1442 \mathrm{~ms}$ and $1703 \mathrm{~ms} ; p<0.01$ ). Accuracy for the tasks 1 and 4 was significantly better for the digital (Task 1: mean distance 0, $Z=2.67, p=0.008, r=0.44$; Task 4: mean distance $0, Z=3.06$, $p=0.002, r=0.51$ ) and for the redundant speedometer (Task 1: mean distance $0, Z=2.67, p=0.008, r=0.44$; Task 4: mean distance $0, Z=3.06, p=0.002, r=0.51$ ) than for the analogue speedometer (Task 1: mean distance 0.26 ; Task 4: mean distance 0.51).

For the task 2, the one-way repeated measures ANOVA showed a significant effect of the speedometer $\left(F(2,34)=50.72, p<.001, \eta_{\mathrm{p}}{ }^{2}=0.75\right)$ showing that the analogue speedometer $(1092 \mathrm{~ms})$ implied longer task completion times than the digital $(827 \mathrm{~ms} ; p<0.01)$ and the redundant speedometer $(921 \mathrm{~ms} ; p<0.01)$. These results were not found for the task $\mathbf{5}$, for which no significant different was found for pairwise comparisons of speedometers $(1177 \mathrm{~ms}$ for the digital, $1100 \mathrm{~ms}$ for the analogue, and $1189 \mathrm{~ms}$ for the redundant). The $3 \times 2$ repeated-measures ANOVA revealed significant main effects of the speedometer $(F(2,34)=18.57, p<.001$, $\left.\eta_{\mathrm{p}}{ }^{2}=0.23\right)$, the tasks $\left(F(1,17)=33.29, p<.001, \eta_{\mathrm{p}}{ }^{2}=0.72\right)$, and the two-way interaction $(F(2,34)=35.90, p<.001$, $\left.\eta_{\mathrm{p}}{ }^{2}=0.67\right)$. Tukey HSD post-hoc test showed that task completion times were significantly longer for the task 5 than for the task 2 for with the digital speedometer (Task 2: 827ms, Task 5: 1177ms; $p<.001$ ) and with the redundant speedometer (Task 2: $921 \mathrm{~ms}$, Task 5: 1189ms; $p<.001$ ). 
For the task 3, the effect of speedometer was significant $\left(F(2,34)=117.25, p<.001, \eta_{\mathrm{p}}{ }^{2}=0.87\right)$. The task completion times were significantly higher with the digital speedometer $(874 \mathrm{~ms})$ than with the analogue (989ms; $p<.001)$ and the redundant speedometer (1034ms; $p<.001)$.

For the task 6, no significant difference of task completion times was found $(9446 \mathrm{~ms}$ for the digital, $2598 \mathrm{~ms}$ for the analogue, and $2229 \mathrm{~ms}$ for the redundant; $\left.F(2,34)=3.07, p=0.059, \eta_{\mathrm{p}}{ }^{2}=0.15\right)$. Nevertheless, accuracy was significantly better with the analogue speedometer (mean distance: $315 \mathrm{~ms}$ ) than with the digital speedometer (mean distance: $568 \mathrm{~ms} ; Z=3.64, p<.001, r=0.61$ ).

\subsection{Usability}

(insert Fig.7)

Pairwise comparisons were led for each dimension of the questionnaire for the three speedometers using Wilcoxon tests. On four of the five dimensions (Fig. 7), the redundant speedometer was scored significantly better than the analogue speedometer (efficiency: respectively 4.67 and 3.61, $Z=3.01, p=0.003, r=0.50$; memorability: respectively 4.83 and $4.28, Z=2.34, p=0.019, r=0.39$; errors: respectively 4.61 and 3.39 , $Z=2.97, p=0.003, r=0.50$; and satisfaction: respectively 4.44 and $3.56, Z=3.18, p=0.001, r=0.53$ ). Memorability scores were significantly higher for the redundant speedometer than for the digital speedometer (respectively 4.83 and $4.44 ; Z=1.96, p=0.050, r=0.33$ ). Drivers scored higher the analogue speedometer for the willingness to errors than the digital speedometer (respectively 3.39 and 4.22; $Z=2.10, p=0.036, r=0.35$ ). Regarding the order of preference, 13 drivers on 18 ranked the redundant speedometer at the first place (mean rank of preference: 1.39/3), and the other 5 chose the digital speedometer (mean rank of preference: $2.06 / 3$ ). The analogue speedometer was ranked either at the second or third place (mean rank of preference: $2.56 / 3$ ). Similarly, the satisfaction scores (between 0 and 10) were significantly better for the redundant speedometer than for the analogue speedometer (respectively 8.17 and 6.06; $Z=3.35, p<.001, r=0.56$ ).

\section{Discussion}

First, this study aimed at investigating whether the existing results in the literature comparing analogue and digital speedometers were still valid for truck driving. When reading the speed value (task 1), the digital speedometer was found to be more efficient, less visually distracting, and more accurate, when compared to the analogue speedometer. This difference is notable, as the time spent with the eyes off the road was reduced by more than $700 \mathrm{~ms}$. The reading was completed about $650 \mathrm{~ms}$ faster $(795 \mathrm{~ms}$ against $1442 \mathrm{~ms})$. Similarly, in the second task, where the participant was asked to tell if the speed value was over or under $50 \mathrm{~km} / \mathrm{h}$ (task 2 ), clear advantages were found when using the digital speedometer. More specifically, there was a gain of $360 \mathrm{~ms}$ of total glance duration on the road, and the task completion time was reduced by $230 \mathrm{~ms}$. On the contrary, to tell if the speed is decreasing or increasing (task 3), the analogue speedometer was found to be more appropriate, as there was a gain of $313 \mathrm{~ms}$ of eyes on the road and the task completion time was $449 \mathrm{~ms}$ shorter. These results, which are dependent on the task being performed, are completely in line with the previous literature (Ishii, 1980; Simmonds et al., 1981; Haller, 1991; Green, 1988; Castro \& Horberry, 2004; Kiefer \& Angell, 1993; Walter, 1991). The differences between both speedometers are even more pronounced in this study. More specifically, the digital speedometer minimised the time spent with the eyes-off-road from $703 \mathrm{~ms}$ in this study, as compared to $70 \mathrm{~ms}$ in the study conducted by Ishii (1980). The reasons behind this phenomenon were not examined in the literature, but some assumptions were mentioned, in order to explain the advantages of the analogue speedometer when performing a dynamic reading task. An analogue speedometer would provide more speed information in the driver's peripheral vision (Kiefer \& Angell, 1993). Thus, the driver would anticipate the pointer position and would get information based on the angle and the movement of the pointer, before having the display in their central vision. Moreover, the analogue speedometer benefits from the dynamic and continuous display of the speed information (Kiefer \& Angell, 
1993). This continuous information stream allows drivers to know whether the vehicle is accelerating or decelerating without them having to read the exact numerical value. Rather, it provides spatial cues using the dynamic pointer which would help the driver recognise the change in rate and the direction of the change (Castro \& Horberry, 2004). However, the digital speedometer is highly dependent of the rate at which it refreshes. Therefore, the information is not continuous and this could impact dynamic reading. These process differences vary depending on the task being performed, and can be related to the skill-rules-knowledge model of Rasmussen (1983). With an analogue speedometer, detecting a dynamic speed change would involve relying on sensory-motor capabilities, which correspond to skill-based performance. Indeed, the analogue speedometer would provide signals that correspond to continuous sensory inputs, which require no reference to any known content associated with the reading task. Rather, with a digital speedometer, the dynamic speed change process would be more complex and would rely on rules. In fact, the driver has to determine if the first speed value is superior or inferior to the second speed value read, in order to conclude if the vehicle is decelerating or accelerating. The use of a procedure known as a rule-based level would match the fact that the task is more expensive with the digital speedometer, than it is with the analogue speedometer. Likewise, to read a speed value with a digital speedometer would be knowledge-based, as would the analogue speedometer. In the usability questionnaire, drivers reported that they could make more errors with the analogue speedometer than they could with the digital speedometer. This result could be due, in major part, to the questions associated with the first task, for which accuracy was better for the digital speedometer. For this task, even if both speedometers were acting like signs, to directly read the value would be less costly than to see the position of the pointer between the graduated level, and then, to interpret the value. To compare the speed value with a target speed, both types of speedometers act like signs, with their readings referring to a rule.

The second objective of this study was to assess the usability, efficiency and visual distraction associated with a redundant speedometer. Results highlighted that the redundant speedometer was effective for the three reading types (i.e. absolute, relative and dynamic). For the task 1 (read the absolute speed value), the redundant speedometer was found to be more efficient, less visually distracting, and more accurate than the analogue speedometer (similar to the digital speedometer performances). The difference between both speedometers resulted in a reduction in the amount of time spent with eyes off of the road by more than $600 \mathrm{~ms}$, and would correspond to a driving distance of $15 \mathrm{~m}$ with eyes off of the road when travelling at $90 \mathrm{~km} / \mathrm{h}$. For the task 2 (tell if the speed value is under or over $50 \mathrm{~km} / \mathrm{h}$ ), the redundant speedometer was also more efficient and less visually distracting than the analogue speedometer. The magnitude of this effect was lower than for the task 1. More specifically, the task was completed about $170 \mathrm{~ms}$ faster, and the time spend with eyes off of the road decreased by about $230 \mathrm{~ms}$. Moreover, the redundant speedometer was more visually distracting than the digital speedometer, with a difference of approximately $131 \mathrm{~ms}$. For the task 3 (say if the speed is decreasing or increasing), the redundant speedometer was more efficient and less visually distracting than the digital speedometer (gain about $250 \mathrm{~ms}$ ). Subjectively speaking, the redundant speedometer scored better than the analogue speedometer on four of the usability dimensions. The four usability dimensions are efficiency, memorability, errors, and satisfaction. In addition, the redundant speedometer scored better than the digital speedometer on the memorability dimension. The findings outline the fact that presenting both speedometer types at the same time did not create interference. Furthermore, it performed as well as the best of each individual type for the tasks 1 and 3. For the task 2, the redundant display produced a longer glance duration when compared to the digital speedometer. However, the gap between the redundant speedometer and the digital speedometer was lower $(131 \mathrm{~ms})$ than the gap between the redundant speedometer and the analogue speedometer $(230 \mathrm{~ms})$. On average, for the three tasks, the redundant speedometer implied shorter glance duration (1178ms) than both other speedometers (12219ms for the digital; $1444 \mathrm{~ms}$ for the analogue). Wickens and Gosney (2003) proposed a five-category classification of redundant presentation effects for secondary tasks. This result matches with the 'best of both worlds' pattern, as it describes a redundant display that produced performance equal to or better than the two single conditions. The higher visual clutter does not appear to create a perceived interference, and we can assume that, until an upper limit to the amount of information presented, it would be similar for an entire instrument cluster. In fact, Yoon et al. (2015) showed that the perceived complexity of the speedometer is correlated with the perceived complexity of the whole instrument cluster. Similarly, the repetition of the two speed information system did not created a negative redundancy effect as in instructional design (Kalyuga et al., 2003). The human-machine cooperation model of Navarro (2016) describes the different steps associated to human processing while interaction with a human- 
machine interface. We can assume that the use of a redundant speedometer would improve perception (attention and perception module in the model), as the total glance duration is reduced. However, it would not impact cognitive processing (situation diagnosis and control module) as no effect was reported on glance frequencies. We can attribute these results to different explanations. First, the fact that task completion times are not prolonged for the redundant speedometer, when compared to the single types presented, indicates that the time spent processing the information did not include additional decision time to choose which type to read. Drivers would automatically select the type that they considered to be better for the task. They would have a metacognitive knowledge of the type chosen for each type of reading, such as looking at the analogue type to detect a dynamic speed change, and looking at the digital type to perform an absolute reading of the speed. Kalyuga et al. (2003) showed that expertise can play a role when processing redundancy. It would be interesting to investigate if this metacognitive knowledge could be linked to truck drivers' expertise, by assessing a redundant speedometer with a novice driver or someone who does not drive at all. Second, Baber and Wankling (1992) argued that the inclusion of redundant information could reduce uncertainty, and therefore reduce decision times. This remaining question could be addressed by evaluating drivers' gaze patterns on the redundant display during the three reading tasks (not performed in this study due to the lack of spatial resolution). Finally, it is important to note that redundancy has a common occurrence in everyday life. Bertelson and De Gelder (2004) stressed that the existence of co-occurring information (whether natural or artificial) create opportunities to improve the performance of perceiving systems. The third objective of this study was to perform an evaluation on more contemporary uses, such as cruise control, and a display that shows the road's speed limit. This was done in order to complement and update previous results. The absolute reading task include additional speed information (task 4) produced results comparable to the task 1 (the same task without additional information). The analogue speedometer was found to be less efficient, more visually distracting, and less accurate than both other speedometers. The presence of additional information on the speedometer significantly impacted the analogue type, as the time to complete the task was lengthened by $260 \mathrm{~ms}$ and the time associated with glance duration away from the road was increased by $229 \mathrm{~ms}$. For the task 5 (determine if the speed is under or over the road speed limit displayed on the speedometer), no difference was found between the three speedometers. Rather, the advantage associated with the digital speedometer and the redundant speedometers for the same relative reading task without system disappeared (i.e. based on a known target speed). The off-road glance duration and task completion times were increased compared to task 2 (350ms longer for the digital and $268 \mathrm{~ms}$ for the redundant). For the task 6 (determine the moment when the actual speed reaches the cruise control target speed), the number of glances was higher with the digital speedometer than it was with the analogue and redundant speedometers. Drivers were also more accurate with the analogue speedometer than they were with the digital speedometer. However, the time spent with eyes off of the road and the task completion times were not significantly different between the three speedometers. Therefore, the superiority of the analogue and redundant speedometers for dynamic reading tasks is still present, but the magnitude of the difference has been greatly reduced. These findings show that the gap between the three speedometers is reduced for longer and more complex tasks (task 5 and 6). Moreover, the increase of distraction and task completion times for the digital and redundant speedometers, when comparing the speed to the road speed limit displayed on the dashboard (task 5), is particularly informative for practitioners. In fact, this intelligent driver assistance system generalizes in cars, and further researches should focus on the more efficient way to display the road speed limit, in order to limit the time in which a driver spends with their eyes off of the road. In this study, the same information was presented in two forms over a single-modality, with the modality being vision. Findings showed that the redundant presentation of both speedometer types did facilitate task completion, without degrading the processing of information. However, according to the multiple resource theory (Wickens, 2002), vision resources are limited and can be overloaded. Vision is the sensorial modality that is most used while driving (Sivak, 1996). To solicit another modality could result in the freeing of resources used for the management of visual information related to the primary driving task. For example, it would be interesting to explore a cross-modal redundant speedometer. Other studies have successfully explored the introduction of multimodal interfaces. For example, adding in auditory feedback could improve the amount of visual attention that the driver attributes to the driving scene (to menu navigation for a secondary interface: Tardieu et al., 2015; for an ADAS interface: Houtenbos et al., 2017; for warning interfaces: Biondi et al., 2017) or improve the driver's satisfaction level (Jakus et al., 2015). For our topic, multimodal speedometers have been investigated (Yang \& Ferris, 2016) and other designs of visually redundant speedometers were explored, such as speedometers that mix a digital display and ambient colour (Ustwo, 2016). Findings concluded that performances were good for ambient-visual, auditory, and 
tactile speedometer displays, and that redundancy with an auditory display, such as beat pattern, was beneficial (Yang \& Ferris, 2016). Another solution might be to investigate dynamic interfaces, in order to provide additional relevant information (May, 2013; Davidsson \& Alm, 2014). This perspective would be particularly suitable for displaying additional speed information, such as displaying the speed limit information only when the speed limit is broken. Indeed, results show that performance was reduced when this information was added. Alternative design solutions for trucks could also be explored in future research.This study contributes to the current knowledge on this topic, by adding data on redundant speedometers and deepening knowledge on contemporaneous use cases. However, future research should address further issues. First, naturalistic research would be necessary to complement this exploratory study. Even if gaps exist between the three speedometers, which are reduced for more complex tasks using speed regulation systems, this paper presents promising findings in favour of redundant speedometer displays. Kiefer and Angell (1993) reported that an analogue speedometer was more effective for the detection of pedestrians than a digital one. More specifically, on average, $18 \%$ of pedestrians were not detected with an analogue speedometer, while an average of $22 \%$ of pedestrians were not detected when using digital speedometer. Further research could focus on situational awareness with redundant speedometers, as compared to the traditional speedometer types. Indeed, even if it is essential to prevent overload (i.e. unnecessary high mental workload) while interacting with a speedometer, underload can be detrimental to performance just as it is to mental overload (Young et al., 2015). Besides, the methodological framework of this study lacked ecological validity. More specifically, speedometers were used on a task-based protocol system, and not in natural driving. Indeed, the desire was to isolate performance for each of the three tasks, which would have been mixed during natural driving scenarios. It would be interesting to find similar results for real driving scenarios, when a speedometer always displayed. A naturalistic study would also be beneficial in bringing in objective data, which will assist with the interpretation of these findings. In this study, speedometers were assessed individually on each of the three reading tasks. In a vehicle, the same speedometer is used for the three different reading tasks. As such, it would be necessary to evaluate which proportion of each reading type is used, in order to conclude which is the best speedometer.

\section{Conclusions}

The design of the speedometer is directly linked to safety considerations. It is essential to provide input to practitioners on the following objectives: quickly providing accurate speed information and minimising time spend with eyes off of the road. With the development of screen instrument clusters and speed regulation systems, the human factors literature presents only limited answers to contemporary concerns. This experiment updated previous literature on analogue and digital speedometers, by confirming task-dependant results for screen instrument clusters. The digital speedometer is more efficient and less visually distracting for absolute and relative reading tasks, whereas the analogue speedometer is more effective for detecting a dynamic speed change. The redundant speedometer has the best performance when compared to the two single types for each of the three reading tasks. Even if gaps between the three speedometers are reduced for more complex tasks using speed regulation systems, this paper presents promising findings in favour of redundant speedometer displays. The findings of this paper meet both theoretical and applied knowledge, have direct design implications for truck dashboards, and contribute to the theoretical work on redundant information processing.

\section{Acknowledgement}

This work was performed within the framework of the LABEX CORTEX (ANR-11-LABX-0042) of University of Lyon, within the program 'Investissements d'Avenir' (ANR-11-IDEX-0007) operated by the French National Research Agency (ANR). This work has also been made possible through a CIFRE PhD convention from the ANRT (ANRT-2013/1405), funded by the French Ministry of Higher Education and Research. The authors wish to thank Ghislaine Goullioud for the support and useful inputs during the design of the materials. 


\section{References}

Baber, C., \& Wankling, J. (1992). An experimental comparison of test and symbols for in-car reconfigurable displays. Applied Ergonomics, 23(4), 255-262.

Bedinger, M., Walker, G. H., Piecyk, M., \& Greening, P. (2015). 21st century trucking: A trajectory for ergonomics and road freight. Applied Ergonomics, 53, 343-356.

Bertelson, P., \& De Gelder, B. (2004). The psychology of multimodal perception. Crossmodal space and crossmodal attention, 141-177.

Biondi, F., Strayer, D. L., Rossi, R., Gastaldi, M., \& Mulatti, C. (2017). Advanced driver assistance systems: Using multimodal redundant warnings to enhance road safety. Applied ergonomics, 58, 238-244.

Castro, C., \& Horberry, T. (2004). The effects of different display types with respect to reading numerical information and detecting speed change. Traffic and Transport Psychology: Theory and Application: Proceedings of the ICTTP 2000, 301-315.

Coury, B. G., \& Pietras, C. M. (1989). Alphanumeric and graphic displays for dynamic process monitoring and control. Ergonomics, 32(11), 1373-1389.

Davidsson, S., \& Alm, H. (2014). Context adaptable driver information - Or, what do whom need and want when?. Applied ergonomics, 45(4), 994-1002.

Federal Motor Carrier Safety Administration. (2014). Large Truck and Bus Crash Facts 2012. FMCSA-RRA14-004. Washington, DC: Department of Transportation.

Green, P. (1988). Human factors and gauge design: a literature review (No. UMTRI-88-37). The University of Michigan Transportation Research Institute.

Haller, R. (1991). Experimental investigation of display reading tasks in vehicles and consequences for instrument panel design. Vision in Vehicles, 3, 197-203.

Houtenbos, M., De Winter, J. C. F., Hale, A. R., Wieringa, P. A., \& Hagenzieker, M. P. (2017). Concurrent audio-visual feedback for supporting drivers at intersections: A study using two linked driving simulators. Applied Ergonomics, 60, 30-42.

Ishii, I. (1980). Comparison of Visual Recognition Time of Analogue and Digital Displays in Automobiles (No. 800354). SAE Technical Paper.

Jakus, G., Dicke, C., \& Sodnik, J. (2015). A user study of auditory, head-up and multi-modal displays in vehicles. Applied ergonomics, 46, 184-192.

Kalyuga, S., Ayres, P., Chandler, P., \& Sweller, J. (2003). The expertise reversal effect. Educational psychologist, 38(1), 23-31.

Kiefer, R. J., \& Angell, L. S. (1993). A comparison of the effects of an analog versus digital speedometer on driver performance in a task environment similar to driving. Vision in Vehicles, 4, 283-290.

Larsson, P., Engström, J., \& Wege, C. (2017). Virtual eye height and display height influence visual distraction measures in simulated driving conditions. Submitted to: 5th International Conference on Driver Distraction and Inattention, Paris, France.

Liu, Y. C. (2001). Comparative study of the effects of auditory, visual and multimodality displays on drivers' performance in advanced traveller information systems. Ergonomics, 44(4), 425-442.

May, A. J. (2013). Using a 'value-added' approach for contextual design of geographic information. Applied ergonomics, 44(6), 895-908.

Navarro, J. (2016). Human-machine interaction theories and lane departure warnings. Theoretical Issues in Ergonomics Science, 1-29. 
Navarro, J., Mars, F., \& Young, M. S. (2011). Lateral control assistance in car driving: classification, review and future prospects. Intelligent Transport Systems, IET, 5(3), 207-220.

Nielsen, J. (1994). Usability engineering. Amsterdam: Elsevier Science Publishers.

Olaverri-Monreal, C., Lehsing, C., Trubswetter, N., Schepp, C. A., \& Bengler, K. (2013). In-vehicle displays: Driving information prioritization and visualization. IEEE Intelligent Vehicles Symposium, 4, 660-665.

Olsen, A. (2012). The tobii i-vt fixation filter. Tobii Technology.

Rasmussen, J. (1983). Skills, rules, and knowledge; signals, signs, and symbols, and other distinctions in human performance models. Systems, Man and Cybernetics, IEEE Transactions on, (3), 257-266.

Simmonds, G. R., Galer, M., \& Baines, A. (1981). Ergonomics of electronic displays (No. 810826). SAE Technical Paper.

Sivak, M. (1996). The information that drivers use: is it indeed 90\% visual?. Perception, 25(9), 10811089.Sweller, J., Van Merrienboer, J. J., \& Paas, F. G. (1998). Cognitive architecture and instructional design. Educational psychology review, 10(3), 251-296.

Tardieu, J., Misdariis, N., Langlois, S., Gaillard, P., \& Lemercier, C. (2015). Sonification of in-vehicle interface reduces gaze movements under dual-task condition. Applied ergonomics, 50, 41-49.

Ustwo (2016). Are we there yet? Thoughts on in-car HMI. https://usweb-cdn.ustwo.com/ustwoproduction/uploads/2016/07/AreWeThereYet_V1.2.pdf (accessed 14.01.2017)

Volvo Trucks (2013). European Accident Research and Safety Report 2013. http://www.volvotrucks.com/SiteCollectionDocuments/VTC/Corporate/Values/ART\%20Report\%202013_15 Odpi.pdf (accessed 14.01.2017)

Walter, W. (1991). Ergonomic information evaluation of analogue and digital coding of instruments in vehicles. Vision in Vehicles, 3.

Wickens, C. D. (2002). Multiple resources and performance prediction. Theoretical issues in ergonomics science, 3(2), 159-177.

Wickens, C. D., \& Gosney, J. L. (2003, October). Redundancy, modality, and priority in dual task interference. In Proceedings of the Human Factors and Ergonomics Society Annual Meeting (Vol. 47, No. 13, pp. 1590-1594). SAGE Publications.

Wickens, C. D., \& Hollands, J.G. (2000). Engineering psychology and human performance (3 ${ }^{\text {rd }}$ Ed.). Upper Saddle River, NJ: Prentice Hall.

Yang, S., \& Ferris, T. K. (2016, September). Measuring Cognitive Efficiency of Novel Speedometer Displays. In Proceedings of the Human Factors and Ergonomics Society Annual Meeting (Vol. 60, No. 1, pp. 19411945). SAGE Publications.

Yoon, S. H., Lim, J., \& Ji, Y. G. (2015). Assessment model for perceived visual complexity of automotive instrument cluster. Applied Ergonomics, 46, 76-83.

Young, M. S., Brookhuis, K. A., Wickens, C. D., \& Hancock, P. A. (2015). State of science: mental workload in ergonomics. Ergonomics, 58(1), 1-17. 